\title{
Combination of Alpha Lipoic Acid and Superoxide Dismutase Leads to Physiological and Symptomatic Improvements in Diabetic Neuropathy
}

Fulvio Bertolotto and Antonino Massone

Neurophysiology Laboratory, Spine Unit at Santa Corona Hospital, Pietra Ligure, Savona, Italy

Abstract

\begin{abstract}
Background and Objective: The management of diabetic neuropathy is still a challenge for physicians. The aim of this study was to assess the efficacy of a new combination of alpha lipoic acid and superoxide dismutase for the treatment of diabetic neuropathy.

Methods: The setting of this study was ambulatory (outpatient) care. A prospective, non-randomized, open-label study was conducted in 50 patients with diabetes mellitus and with a deficit in both motor and sensory nerve conduction. Treatment was with a new combination of alpha lipoic acid and superoxide dismutase (ALA600SOD ${ }^{\circledR}$ ) for 4 months. Electroneurographic parameters and perceived pain were assessed at baseline and after treatment. Results: After 4 months of treatment, patients significantly $(p<0.001)$ improved their electroneurographic parameters and their perception of pain. Best improvements were observed in sensory nerve conduction.

Conclusion: The combination of two powerful antioxidant agents leads to improvement in both subjective and objective parameters in patients with diabetic neuropathy. New profitable directions for investigations are opened for a non-invasive treatment of diabetic neuropathy in the future.
\end{abstract}

\section{Introduction}

Neuropathy is a microvascular complication of diabetes mellitus that leads to considerable morbidity and a decreased quality of life. ${ }^{[1,2]} \mathrm{Di}$ abetic neuropathy (DN) is a term indicating all signs and symptoms of peripheral nerve dysfunction in diabetic patients in whom other causes of neuropathy have been excluded ${ }^{[3,4]}$ and it is a major public health problem, affecting approx- imately $13-26 \%$ of diabetic patients. ${ }^{[5-9]}$ Conduction studies help to identify and localize focal lesions in a nerve by demonstrating localized slowing down or conduction block. In fact, electrophysiological testing plays an important role in detecting, characterizing and measuring DN. Nevertheless, assessing the severity of painful symptoms and the nerve conduction slowing down is important not only for diagnosis but also to assess the benefits of treatment. 
The understanding that oxidative stress is a unifying mechanism for the cellular pathways that lead to diabetes complications strongly indicates the use of antioxidants in therapies aimed at the prevention of diabetes and the potential reversal of its complications..$^{[10,11]}$ The data so far suggest a number of therapeutic strategies. ${ }^{[9,11,12]}$ According to a recent review, ${ }^{[13]}$ at least 50 molecular formulations have reached the clinical stage of development. Although several analgesic therapies are available to alleviate the symptoms of diabetic neuropathic pain, few options are available to eliminate the root causes and DN remains a challenge for physicians. ${ }^{[14]}$

In animal studies, alpha lipoic acid (ALA) has been shown to prevent or even reverse hyperglycemia-induced nerve dysfunction by reducing free-radical-mediated oxidative stress. ${ }^{[15]}$ It has also been demonstrated that ALA improves nerve blood flow and peripheral nerve fiber conduction and increases endoneurial glucose uptake and energy metabolism in experimental diabetic peripheral neuropathy. ${ }^{[16]}$ Two meta-analyses of randomized, placebo-controlled trials using ALA infusions of $600 \mathrm{mg}$ intravenously/orally per day for 3 weeks in diabetic patients with positive symptoms of peripheral neuropathy have been published ${ }^{[1,17]}$ and suggest that this treatment produces clinically significant improvements in neuropathic symptoms and deficits. When given intravenously, ALA leads to a significant and clinically relevant reduction in neuropathic pain. Improvements with oral administration are less described but strongly marked after just 2 weeks of treatment. ${ }^{[9]}$ Nevertheless, there are a lack of significant data on the effects of ALAs on nerve conduction velocity.

Superoxide dismutase (SOD) is an essential, ubiquitous enzyme that detoxifies highly reactive $\mathrm{O}_{2}^{-}$by catalysis into $\mathrm{H}_{2} \mathrm{O}_{2}$, which in turn is reduced in $\mathrm{H}_{2} \mathrm{O}$ in the mitochondria by glutathione peroxidase and catalase. ${ }^{[18]} \mathrm{SOD}$, which has the important role of neutralizing superoxide radicals, is reduced in diabetic peripheral nerve tissue, thus compounding any enhancement of free radical formation..$^{19-21]}$ Furthermore, SOD has a key role in inhibiting inflammatory response, which is closely correlated with attenuation of hyperalgesia. ${ }^{[22]}$
Since oxidative stress is enhanced in diabetic patients with neuropathy, ${ }^{[12]}$ a pharmacologic strategy aimed at overcoming the deficit of antioxidant agents should provide significant relief from complications for neuropathic patients. The ideal treatment should prevent or arrest the progressive loss of nerve functionality and improve symptoms with minimal side effects.

A new oral formulation combining ALA and SOD, two powerful antioxidant agents singly active in $\mathrm{DN}$, has been proposed as a powerful tool in the treatment of DN.

The aim of this pilot study was to assess changes in nerve conduction velocity and symptomatology in patients with DN treated daily for 4 months with a combination of ALA and SOD.

\section{Patients and Methods}

From May to November 2010, a prospective, non-randomized, open-label, pivotal study was conducted.

The study population included patients with diabetes and with diabetic symmetric sensorimotor polyneuropathy. ${ }^{[23]}$ Patients were treated orally for 4 months with ALA $600 \mathrm{mg}$ and SOD $140 \mathrm{IU} /$ day (ALA600 SOD ${ }^{\circledR}$, Alfa Wassermann, Bologna, Italy).

Data on sex, age and type of diagnosis were collected. Visual analog scale (VAS) was used at baseline and at the end of the 4-month treatment. Electroneurography parameters were assessed by a Dantec (Dantec, Skovlunde, Denmark) keypoint device to collect the signal and for the recording of the responses. The subjects were seated in a comfortable chair and instructed to be as relaxed as possible. Electroneurography parameters included motor nerve (peroneal) conduction and sensory (sural) nerve conduction.

Differences between baseline and post-treatment values were recorded for all measured variables.

All patients were notified of the investigational nature of this study and gave their written informed consent. The study was approved by the institutional review board in accordance with institutional guidelines, including the Declaration of Helsinki. Any adverse event that occurred during the study period was recorded. 
Results are reported as descriptive statistics. Quantitative parameters are reported as mean, minimum, maximum and standard deviations; qualitative parameters are reported as absolute and relative frequencies.

Student's t-test for paired data and Wilcoxon's signed-rank test were used. To assess the difference between sub groups a Mann Whitney-U test and a Fisher's exact test were performed.

$\mathrm{p}$-Values were considered statistically significant if $<0.05$. Statistical analyses were performed with SPSS Statistical Package, version 15.0 (IBM, Armonk, NY, USA).

\section{Results}

Fifty patients affected by DN among outpatients attending the clinic of Unità Spinale dell'Ospedale Santa Corona di Pietra Ligure, Savona, Italy, were prospectively and consecutively enrolled. All the subjects had had type 2 diabetes since 1999 and were treated for this pathology. Twelve patients were discarded due to lacking data or missing follow-up. In two patients no efficacy data were available, ten patients were lost to follow-up due to intercurrent diseases or noncompliance. The final dropout rate was $24 \%$. In the final sample there were 38 patients valuable for the purpose of this study: 17 females and 21 males with a median age of 68.2 years $( \pm 7.4)$, all with diabetes and with a deficit in nerve velocity conduction (diabetic symmetric sensorimotor polyneuropathy). ${ }^{[23]}$

All measured variables were tested for sex differences due to sex dimorphism suggested by clinical observation. In fact, nerve conduction abnormalities have been previously reported as more frequent and severe in males, while neuro- pathic pain and negative sensory symptoms seem to be more frequent in female patients. ${ }^{[24,25]}$ No statistically significant differences were observed between sexes in our patients, thus we report results for the whole sample.

All the measured characteristics significantly improved after treatment ( $<<0.001$, table I). The nerve conductions, both motor and sensory, increased and perceived pain improved. The rate of increment of conduction velocity is greater in the sensory nerve $(12.1 \%)$ than in the motor nerve $(7.1 \%)$. We labelled a good improvement particularly in sensory nerve conduction; most of the patients had an increment $\geq 10 \%$.

Less than $10 \%$ of patients retained stable measurements in each of the three parameters and worsening of the measured parameters was not observed in any patient (figure 1).

Fifty patients were used for safety analysis and no adverse event occurred during the study.

\section{Discussion}

$\mathrm{DN}$ is a neuropathic disorder that is associated with diabetes. This condition is thought to result from diabetic microvascular injury involving small blood vessels that supply the nerves (vasa nervorum). After all, DN is a degenerative pathology with a progressively disabling course, affecting all peripheral nerves: pain fibers, motor neurons, and autonomic nerves. ${ }^{[26]}$ It can therefore affect all organs and systems since all are innervated. Though therapies are available to alleviate the symptoms of $\mathrm{DN}$, few options are available to eliminate the root causes. The immense physical, psychological, and economic costs of DN underline the need for causally targeted therapies. In fact, causal treatments aim at slowing down pathology

Table I. Parameters pre- and post-treatment with variation in the whole sample ${ }^{\mathrm{a}}$

\begin{tabular}{lllll}
\hline Variable & Pre-treatment & Post-treatment & Variation & p-Value \\
\hline VAS (score) & $5.5 \pm 0.6(5-7)$ & $4.5 \pm 0.6(4-6)$ & $-1.0 \pm 0.4(-2-0)$ & $<0.001$ \\
MNC (m/sec) & $36.6 \pm 2.9(30-40)$ & $39.2 \pm 3.5(32-45)$ & $2.5 \pm 1.6(0-7)$ & $<0.001$ \\
SNC (m/sec) & $32.3 \pm 6.2(22-45)$ & $36.2 \pm 6.1(25-48)$ & $3.9 \pm 2.2(0-12)$ & $<0.001$ \\
\hline
\end{tabular}

a Data expressed as mean \pm SD (range).

$\mathbf{M N C}=$ motor nerve conduction; $\mathbf{S N C}=$ sensory nerve conduction; $\mathbf{V A S}=$ visual analog scale. 


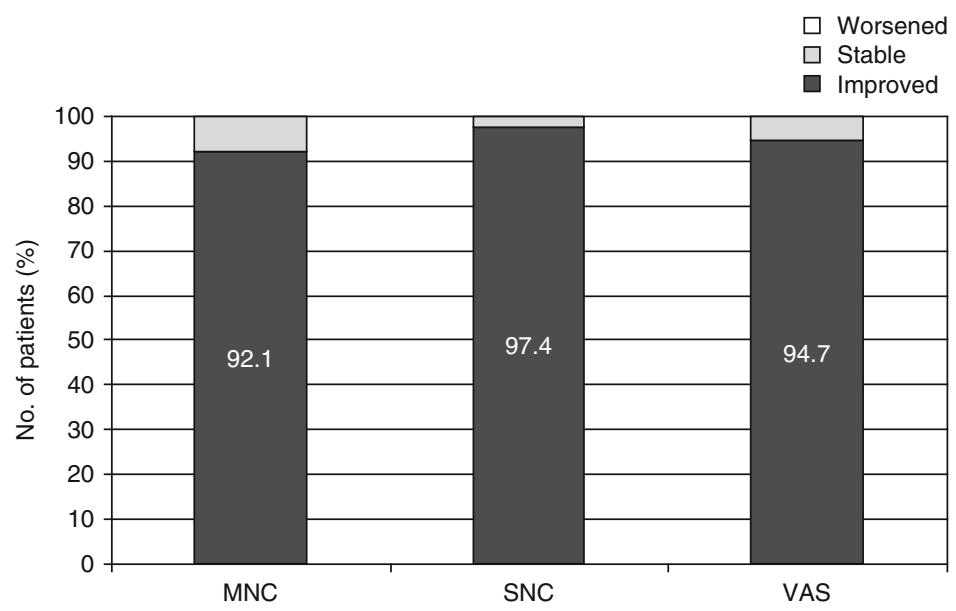

Fig. 1. The percentages of patients that improved, remained stable or worsened in the measured parameters after treatment. MNC $=$ motor nerve conduction; SNC = sensory nerve conduction; VAS = visual analog scale.

progression besides reducing use of analgesics and improving nerve deficits. ${ }^{[27]}$

ALA is a powerful antioxidant and several studies - including the SYDNEY2 trial - have demonstrated an improvement in neuropathic symptoms and deficits. ${ }^{[9]}$ Results of a metaanalysis ${ }^{[28]}$ provided evidence that treatment with ALA $600 \mathrm{mg} /$ day over 5 weeks is safe and significantly improves both neuropathic symptomatology and neuropathic deficits to a clinically meaningful degree in diabetic patients with symptomatic polyneuropathy.

SOD protects nerves from injury in cell culture and in animal models of DN. ${ }^{[29]}$ Direct activity on nerve fibers exposed to oxidative stress and indirect activity targeting vasa nervorum make SOD a powerful adjuvant tool in the treatment of DN.

Our diagnostic group aims to detect specific sensory profiles through clinical examination, questionnaires dedicated to neuropathic pain and laboratory tools.

A new oral formulation combining ALA and SOD was investigated in this prospective pilot study, through assessment of changes in nerve conduction velocity and patients' symptomatology.

Previous studies reported that one potential limitation of the standard electrophysiological techniques is in detecting therapeutic benefit. Our study stated that the improvement of nerve con- duction velocity (objective data) matches the improvement of perceived pain in diabetic patients (subjective data). Furthermore, we observed a greater improvement in the sensory nerve conduction than in the motor conduction, which could suggest a preferential action of the ALA and SOD formulation that may indicate a future direction of investigation.

The synergistic action of ALA and SOD improves both nerve conduction velocity and perceived pain, stating few or absent side effects of this formulation and of the two single components already confirmed by clinical and postmarketing surveillance. ${ }^{[17,30]}$ SOD prevents the formation of free radicals and ALA promotes their removal; furthermore, the oral formulation (with improved bioavailability) improves the patient's quality of life, removing the burden of infusion therapy. In addition, this neurotrophic integrator shows clinically relevant results over a brief time period with homogeneous improvements amongst patients.

We report the present study as a clinical experience because we chose a per protocol analysis to maximize the opportunity for the proposed treatment to show its efficacy and the actual number of enrolled patients was relatively small as a prospective study. Further studies (e.g. a phase III, multicenter trial with a group treated with ALA and SOD vs a group treated with placebo) 
are warranted to support our results with a greater sample size and to investigate placebo effects and longer follow-up for duration of response and for treatment safety. Furthermore, future research should quantify the added value of SOD over ALA.

\section{Conclusion}

Our study is the first to show that treatment with a combination of ALA and SOD leads to an improvement both in symptomatology and in electroneurographic parameters in patients affected by DN.

The results suggest a new scenario for the management of $\mathrm{DN}$, a new non-invasive treatment with no registered adverse events. This pivotal study indicates future directions for useful investigation.

\section{Acknowledgments}

No sources of funding were used in the study design, collection, analysis, or interpretation of the data, or in writing this article. The authors declare that they have no conflicts of interest to disclose.

\section{References}

1. Mijnhout GS, Alkhalaf A, Kleefstra N, et al. Alpha lipoic acid: a new treatment for neuropathic pain in patients with diabetes? Neth J Med 2010; 68 (4): 158-62

2. Van Acker K, Bouhassire D, De Bacquer D, et al. Prevalence and impact on quality of life of peripheral neuropathy with or without neuropatic pain in type 1 and type 2 diabetic patients attending hospital outpatients clinics. Diabetes Metab 2009; 35: 206-13

3. Boulton AJ, Vinik AI, Arezzo JC, et al. Diabetic neuropathies: a statement by the American Diabetes Association. Diabetes Care 2005; 28: 956-62

4. Vallianou N, Evangelopoulos A, Koutalas P. Alpha-lipoic acid and diabetic neuropathy. Rev Diabet Stud 2009; 6 (4): 230-6

5. Daousi C, Benbow SJ, Woodward A, et al. The natural history of chronic painful peripheral neuropathy in a community diabetes population. Diabet Med 2006; 23: 1021-4

6. Davies M, Brophy S, Williams R, et al. The prevalence, severity and impact of painful diabetic peripheral neuropathy in type 2 diabetes. Diabetes Care 2006; 29: 1518-22

7. Greco D, Bambina F, Pisciotta M, et al. Clinical characteristics and associated comorbidities in diabetic patients with cranial nerve palsies. J Endocrinol. Epub 2011 Mar 7

8. Gries FA, Cameron NE, Low PA, et al. Textbook of diabetic neuropathy. Stuttgart: Thieme, 2003

9. Ziegler D, Ametov A, Barinov A, et al. Oral treatment with alpha-lipoic acid improves symptomatic diabetic poly- neuropathy: the Sydney 2 Trial. Diabetes Care 2006; 29: 2365-70

10. Ceriello A, Testa R. Antioxidant anti-inflammatory treatment in type 2 diabetes. Diabetes Care 2009; 32 Suppl. 2: S232-6

11. Vincent AM, Edwards JL, Sadidi M, et al. The antioxidant response as a drug target in diabetic neuropathy. Curr Drug Targets 2008; 9: 94-100

12. Ziegler D, Sohr CGH, Nourooz-Zadeh J. Oxidative stress and antioxidant defense in relation to the severity of diabetic polineuropathy and cardiovascular autonomic neuropathy. Diabetes Care 2004; 27 (9): 2178-83

13. Gilron I, Coderre TJ. Emerging drugs in neuropathic pain. Expert Opin Emerg Drugs 2007; 12: 113-26

14. Cruccu G, Sommer C, Anand P, et al. EFNS guidelines on neuropathic pain assessment: revised 2009. Eur J Neurol 2010; 17: 1010-8

15. Van Dam PS. Oxidative stress and diabetic neuropathy: pathophysiological mechanisms and treatment perspectives. Diabetes Metab Res Rev 2002; 18 (3): 176-84

16. Low PA, Nickander KK, Tritschler HJ. The roles of oxidative stress and antioxidant treatment in experimental diabetic neuropathy. Diabetes 1997; 46 Suppl. 2: S38-42

17. Ziegler D. Thioctic acid for patients with symptomatic diabetic polyneuropathy: a critical review. Treat Endocrinol 2004; 3 (3): 173-89

18. Wallace DC. Mitochondrial DNA. Methods Mol Biol 2002; 197: 3-54

19. Giugliano D, Ceriello A, Paolisso G. Oxidative stress and diabetic vascular complications. Diabetes Care 1996; 19: 257-67

20. Maier CM, Chan PH. Role of superoxide dismutase in oxidative damage and neurodegenerative disorders. Neuroscientist 2002; 8 (4): 323-34

21. Nourooz-Zadeh J, Tajaddini-Sarmadi J, McCarthy S, et al. Elevated levels of authentic plasma hydroperoxides in NIDDM. Diabetes 1995; 44: 1054-8

22. Wang ZQ, Porreca F, Cuzzocrea S, et al. A newly identified role for superoxide in inflammatory pain. J Pharmacol Exp Ther 2004; 309 (3): 869-78

23. Kimura J. Nerve conduction and needle electromyography. In: Dyck PJ, Thomas PK, editors. Peripheral neuropathy. 4th ed. Philadelphia (PA): Saunders, 2005: 899-969

24. Albers JW, Brown MB, Sima AA, et al. Nerve conduction measures in mild diabetic neuropathy in the Early Diabetes Intervention Trial: the effects of age, sex, type of diabetes, disease duration, and anthropometric factors. Tolrestat Study Group for the Early Diabetes Intervention Trial. Neurology 1996; 46: 85-91

25. Kiziltan ME, Gunduz A, Kiziltan G, et al. Peripheral neuropathy in patients with diabetic foot ulcera: clinical and nerve conduction study. J Neurol Sci 2007; 258: 75-9

26. Shaikh AS, Somani RS. Animal models and biomarkers of neuropathy in diabetic rodents. Indian J Pharmacol 2010; 42 (3): 129-34

27. Edwards JL, Vincent AM, Cheng HT, et al. Diabetic neuropathy: mechanisms to management. Pharmacol Ther 2008; 120: 1-34 
28. Ziegler D, Nowak H, Kempler P, et al. Treatment of symptomatic diabetic polyneuropathy with the antioxidant alpha-lipoic acid: a meta-analysis. Diabet Med 2004; 21: 114-21

29. Vincent AM, Russell JW, Sullivan KA, et al. SOD2 protects neurons from injury in cell culture and animal models of diabetic neuropathy. Exper Neurol 2007; 208 216-27
30. Ziegler D. Painful diabetic neuropathy. Diabetes Care 2009; Suppl. 2 (32): S414-9

Correspondence: Fulvio Bertolotto, MD, Laboratorio di neurofisiologia dell'Unità Spinale dell'Ospedale Santa Corona di Pietra Ligure via XXV Aprile 38, Savona, Italy.

E-mail: fulvio.bertolotto@libero.it 